\title{
Uncertainties in ship-based estimation of waves and responses
}

\author{
Montazeri, Najmeh; Jensen, J. Juncher; Nielsen, Ulrik D.
}

Published in:

Proceedings of OCEANS '15

Publication date:

2015

Document Version

Peer reviewed version

Link back to DTU Orbit

Citation (APA):

Montazeri, N., Jensen, J. J., \& Nielsen, U. D. (2015). Uncertainties in ship-based estimation of waves and responses. In Proceedings of OCEANS '15 IEEE.

\section{General rights}

Copyright and moral rights for the publications made accessible in the public portal are retained by the authors and/or other copyright owners and it is a condition of accessing publications that users recognise and abide by the legal requirements associated with these rights.

- Users may download and print one copy of any publication from the public portal for the purpose of private study or research.

- You may not further distribute the material or use it for any profit-making activity or commercial gain

- You may freely distribute the URL identifying the publication in the public portal

If you believe that this document breaches copyright please contact us providing details, and we will remove access to the work immediately and investigate your claim 


\section{Uncertainties in ship-based estimation of waves and responses}

\begin{abstract}
Najmeh Montazeri
Department of

Mechanical Engineering,

Technical University of Denmark

Email: najmon@mek.dtu.dk

Abstract-Real time estimation of waves and ship responses using onboard measurements has been under investigation in recent years. This has been done using different methods, including parametric and non-parametric models. Since none of the methods are believed to be fully accurate, it is important to assign an uncertainty measure to the waves and responses that are being estimated. In this paper, a parametric model approach based on moments of responses is considered for wave estimation. A method based on linear error propagation is introduced to assess the uncertainty of wave estimations. The uncertainty of response calculation based on the estimated wave is also quantified.
\end{abstract}

J. Juncher Jensen

a) Department of

Mechanical Engineering,
NTNU,Norway

Email: jjj@mek.dtu.dk

bechnical University of Denmark Technical University of Denmark

b) Centre for Autonomous Marine b) Centre for Autonomous Marine Operations and Systems(AMOS), Operations and Systems(AMOS),

NTNU,Norway

Email: udn@mek.dtu.dk

\section{INTRODUCTION}

Onboard prediction of seakeeping performance and structural loads of the ship has been of great consideration in recent years for the goal of operational safety. For this purpose, mathematical models should be able to describe the ship's behaviour in a seaway. Typically, the idea of response prediction is based on combination of the wave estimate and the transfer function of a response to be predicted. The outcome is the reproduced response statistics; often visualized in a graphical user interface. In addition, uncertainty modelling has also been of interest in reliability-based studies; e.g.[1, 2]. There are many aspects that impose uncertainties to wave and response estimations; e.g. measurement data, environmental data, mathematical models, transfer functions and ship data. The following approach can be used to quantify the most important uncertainties.

\section{WAVE ESTIMATION METHOD}

A wave estimation method based on ship responses is considered here, where a JONSWAP model is fitted to obtain the measured responses using the transfer functions. The optimisation of the sea state parameters is carried out through the spectral moments of the responses spectra. For complete description of the estimation procedure, see [3]. The basic cost function for estimation of a simplified short crested unimodal wave spectrum using one specific response can be written as:

$$
R=\int_{0}^{\infty} \int_{-\pi}^{\pi} S(\omega, \theta, H s, T p, \mu) H^{2}(\omega, \theta), d \theta d \omega
$$

$S$ is the JONSWAP wave spectrum which is considered as a function of three main wave parameters: the significant wave height, $H s$, the peak period, $T p$ and the mean wave direction, $\mu$. In this study, the peakedness factor, $\gamma$, and the spreading parameter, $s$, are fixed. $\omega$ is the wave frequency, $\theta$ is the wave direction and $H$ denotes the amplitude of the transfer function. Consequently $R$ gives the 0th spectral moment (or the variance) of the response. 
TABLE I: Ship characteristics

\begin{tabular}{ll}
\hline Properties & Values \\
\hline Overall Length [m] & 349.0 \\
Beam [m] & 42.8 \\
Draft [m] & 14.5 \\
Speed [kn] & 20 \\
\hline
\end{tabular}

TABLE II: The sample wave cases

\begin{tabular}{llllll}
\hline Cases & $H_{s}(\mathrm{~m})$ & $T_{p}(\mathrm{~s})$ & $\mu($ deg. $)$ & $\gamma$ & $s$ \\
\hline A,B (Wind Sea) & 3 & 8 & 45,135 & 1 & 15 \\
C,D (Swell) & 5 & 15 & 45,135 & 4 & 25 \\
\hline
\end{tabular}

\section{CASE STUDY}

The main characteristics of the studied vessel and the operational conditions are given in Table I. Two wind sea and two swell systems are considered as shown in table II where $45 \mathrm{deg}$. and 135 deg. represent stern quartering sea and head quartering sea, respectively. 20 minutes long time histories are simulated for responses using a JONSWAP spectrum and the transfer functions. Since wave records are assumed to be Gaussian processes, the time series are generated using a set of uncorrelated standard normal distributed variables [4]. Three responses: vertical motion at the port side of the midship section, pitch motion and wave induced vertical bending moment at midship section are used for estimation of the sea state. In this study, cross spectral analysis, [3], is not implemented.

\section{LINEAR ERROR PROPAGATION}

Uncertainty analysis can be run by propagating the input uncertainties through the model, all the way to the model output. This can be calculated by the law of linear error propagation $[5,6]$. Assuming that the estimated result, $f$, is a function of $n$ input variables, $x_{n}$, i.e., $f=f\left(x_{1}, x_{2}, \ldots, x_{n}\right)$, the uncertainty in the output, $u_{f}$, can be calculated by:

$u_{f}^{2}=\left(\frac{\partial f}{\partial x_{1}}\right)^{2} u_{x_{1}}^{2}+\left(\frac{\partial f}{\partial x_{2}}\right)^{2} u_{x_{2}}^{2}+\ldots+\left(\frac{\partial f}{\partial x_{n}}\right)^{2} u_{x_{n}}^{2}$

where $u_{x_{n}}$ is the uncertainty in $x_{n}$. Uncertainty analysis assumes the variables to be Gaussian random processes. Thus, all uncertainties $(u)$ are usually considered as standard deviations.
In this paper, for wave estimation part, the above mentioned method is implemented to quantify the error in the outcome of the estimation process where Eq.(1) is taken as $f$.

\section{UNCERTAINTY SOURCES}

Numerical methods for hydrodynamic calculations e.g. strip theory and 3D panel methods have been grown stronger during last decades. However, due to different mathematical models, modelling of boundary value problem, errors of body geometry modelling and inaccurate mass distribution, the transfer function calculations are subjected to both bias (systematic) error and random error. Those errors can be estimated using experiments [6-8]. The bias error can then be entered to the estimation procedure. The randomness of the transfer function, which is focused on here, is usually expressed as:

$$
H(\omega)=\hat{H}(\omega)[1+\epsilon(\omega)]
$$

where $\hat{H}$ is the theoretically calculated transfer function and $\epsilon$ is a zero mean, normally distributed random error with standard deviation $\sigma_{\epsilon}$.

Apart from the transfer functions, the measurements are also subjected to errors. If this error could be approximated by the sensors manufacturer, $R$ in Eq.(1) can be considered random as well. But this error is neglected here. Furthermore, the assumption that the standard wave model (i.e. JONSWAP spectrum here) perfectly represents the actual wave spectrum, is also sceptical. This has lead to wave estimation studies based on nonparametric methods [9-11].

\section{UNCERTAINTY OF SEA STATE ESTIMATION}

Based on the above mentioned assumptions, the variability of the wave spectral ordinate about its mean value, is dependent merely on the transfer functions of the responses considered for estimation and, hence, can be modelled as:

$$
u_{S}^{2}=\left.\frac{1}{K} \sum_{k=1}^{K}\left(\frac{\partial S}{\partial H_{k}}\right)^{2}\right|_{R_{k}=\text { const. }} u_{H_{k}}^{2}
$$

where $K$ is the number of responses and the index $k$ represents the quantities for the $k$ th response. 
Based on Eq.(3) the uncertainty of the $k$ th transfer function can be calculated by:

$$
u_{H_{k}}=\sigma_{\epsilon} \hat{H}_{k}
$$

It should be noted that Eq.(4) assumes the different responses to be statistically uncorrelated. The analytical relation between $S$ and $H_{k}$ is not explicitly available since the parameters contained in $S$ are being estimated through a set of equations based on Eq.(1). Thus, the derivative of $S$ with respect to $H_{k}$ can not be easily calculated.

One way to perform this uncertainty analysis is to treat the transfer functions as a random input (Eq.(3)) to the wave estimation procedure. Uncertainty of the wave parameters can then be obtained from the sampling variance of the estimations. This is quite time consuming since the optimisation algorithm should be run many times.

As an alternative, based on Eq.(1), it can be assumed that the uncertainty of the transfer function is reflected in the magnitude of response variances, i.e. $R$. So Eq. (4) can be replaced by:

$$
u_{S}^{2}=\left.\frac{1}{K} \sum_{k=1}^{K}\left(\frac{\partial S}{\partial R_{k}}\right)^{2}\right|_{H_{k}=\hat{H}_{k}}\left(u_{R_{k}}^{H_{k}}\right)^{2}
$$

where $u_{R_{k}}^{H_{k}}$ represents the error in the response variance due to the error in the transfer function which can be calculated as follows.

Eq.(1) in a discretised form can be expressed as follows for the $k$ th response:

$$
R_{k}=\sum_{j=1}^{m} \sum_{i=1}^{n} S_{i j}\left[\hat{H}_{i j, k}\left(1+\epsilon_{j}\right)\right]^{2}(\delta \omega)^{2}(\delta \theta)^{2}
$$

where $m$ and $n$ are the number of frequencies and the number of directions, respectively. The variance of Eq.(7) yields $\left(u_{R_{k}}^{H_{k}}\right)^{2}$ as follows:

$$
\begin{aligned}
& \left(u_{R_{k}}^{H_{k}}\right)^{2}=\sum_{j=1}^{m} \sum_{i=1}^{n} \\
& S_{i j}^{2}\left[2\left(\sigma_{\epsilon_{j}} \hat{H}_{i j, k}\right)^{4}+4\left(\sigma_{\epsilon_{j}} \hat{H}_{i j, k}\right)^{2} \hat{H}_{i j, k}^{2}\right](\delta \omega)^{2}(\delta \theta)^{2}
\end{aligned}
$$

For simplification, $\sigma_{\epsilon_{j}}$ is considered constant and independent of frequency [8]. $\sigma_{\epsilon}=0.04$, obtained from $[6,7]$ is considered here for all responses. However, this is not true since the accuracy of hydrodynamic calculation of some responses (for instance roll and pitch) are lower than the others (e.g. heave). Taking $\sigma_{\epsilon}$ out of the summations, results in:

$$
\left(u_{R_{k}}^{H_{k}}\right)^{2}=\left(2 \sigma_{\epsilon}^{4}+4 \sigma_{\epsilon}^{2}\right) \hat{R}_{k}^{2}
$$

where $\hat{R}_{k}$ is the variance of the $k$ th response calculated without uncertainties. The partial derivatives in Eq.(6) should be calculated analytically using Eq.(1). $R_{k}$ is considered as a function of the wave parameters. So Eq.(6) can be rewritten as:

$u_{S}^{2}=\frac{1}{K} \sum_{k=1}^{K}\left[\left(\frac{\frac{\partial S}{\partial H s}}{\frac{\partial R_{k}}{\partial H s}}\right)^{2}+\left(\frac{\frac{\partial S}{\partial T_{p}}}{\frac{\partial R_{k}}{\partial T p}}\right)^{2}+\left(\frac{\frac{\partial S}{\partial \mu}}{\frac{\partial R_{k}}{\partial \mu}}\right)^{2}\right]\left(u_{R_{k}}^{H_{k}}\right)^{2}$

where $\frac{\partial S}{\partial H s}, \frac{\partial S}{\partial T p}$ and $\frac{\partial S}{\partial \mu}$ are calculated from JONSWAP spectrum. Following the procedure in Sec. II and [3] the wave parameters of the considered cases are estimated using vertical motion, pitch and vertical bending moment as mentioned before. These parameters are used as input to the JONSWAP spectrum and the uncertainties are calculated from Eq.(10). These uncertainties are expressed as intervals around the spectral ordinates at different directions and frequencies. In other words each spectral ordinate can be expressed as:

$$
S(\omega, \theta)=\hat{S}(\omega, \theta)[1+\zeta(\omega, \theta)]
$$

where $\hat{S}$ is the estimated spectrum and $\zeta$ is the random error with zero mean and standard deviation $\sigma_{\zeta}$. The latter quantity is called the coefficient of variation of the wave spectrum [12] which can be calculated by:

$$
\sigma_{\zeta}=\frac{u_{S}}{\hat{S}}
$$

Figure 1 shows the integrated frequency spectra and the uncertainty with $90 \%$ confidence level based on t-distribution [13]- for the wave cases in Table II. It can be seen that the uncertainty in case A, which is stern quartering wind sea, is quite large for the whole spectrum. However, the variabilities in the other cases are relatively low except in the peak values of the spectra. It could be argued that in case A, the selected set of responses are not optimum for wave estimation. A proper combination of responses can be chosen using sensitivity analysis based on the derivatives in Eq.(6). But this is out of the scope of this paper. 

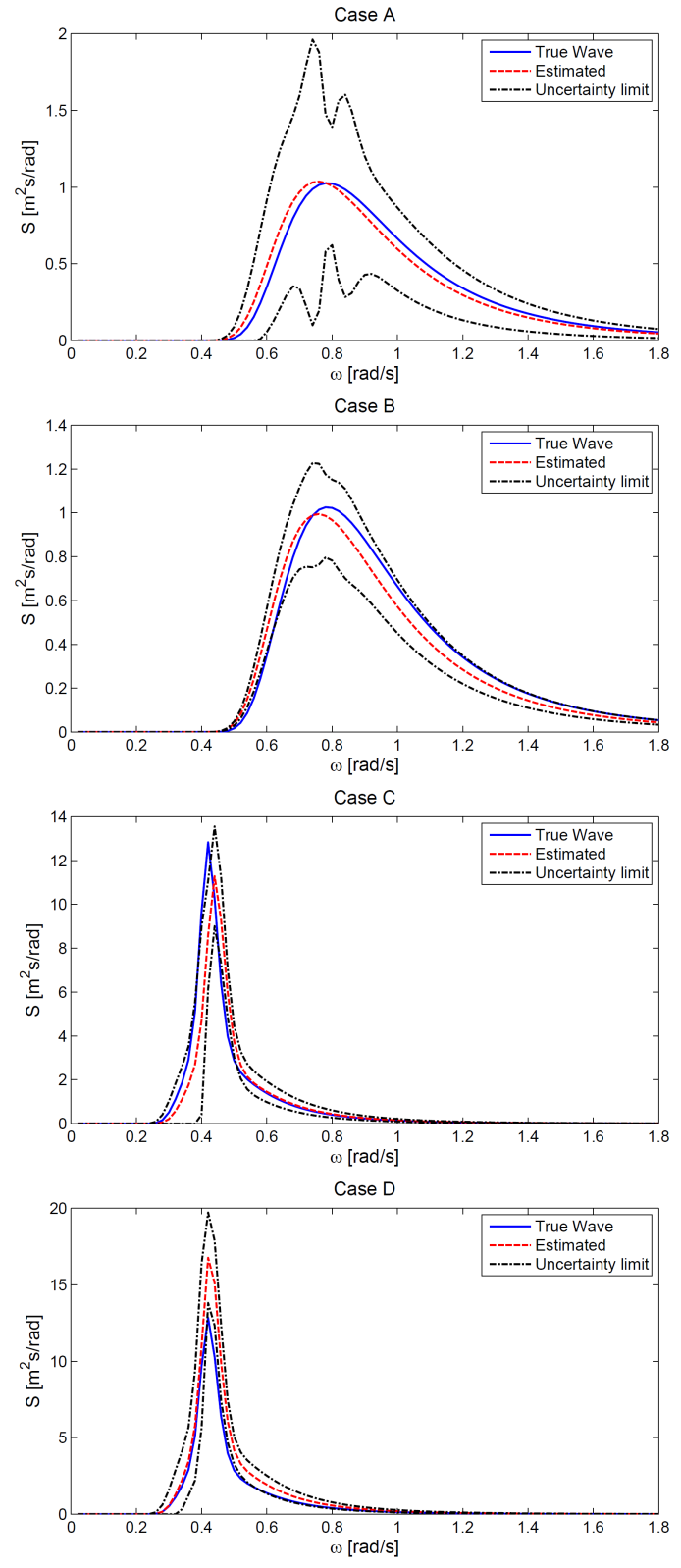

Fig. 1: Wave spectra

\section{UNCERTAINTY OF RESPONSES}

Estimation or prediction of different short-term ship responses in any given operational condition is significant in decision support applications. The energy amount of a response spectra, $R$, is a
TABLE III: Coefficient of variation for different responses

\begin{tabular}{lllll}
\hline Cases & Vertical Motion & Pitch & Roll & VBM \\
\hline A & 0.4 & 0.43 & 0.8 & 0.36 \\
B & 0.24 & 0.27 & 0.17 & 0.21 \\
C & 0.5 & 0.51 & 0.5 & 0.47 \\
D & 0.48 & 0.56 & 0.38 & 0.47 \\
\hline
\end{tabular}

very practical value for description of the response behaviour since it allows different probabilistic statements about the response. For instance, the probability of exceedance is evaluated by Rayleigh distribution as a function of $R$. Thus, the uncertainty of the response is directly related to the uncertainty in $R$ [12]. This value is determined from Eq.(1). So the source of uncertainty of the response calculation refers to both the wave spectrum and the transfer function for a particular response. Therefore, using Eqs.(3) and (11), the statistical expression of $R$ can be written as:

$$
R_{l}=\sum_{j=1}^{m} \sum_{i=1}^{n} \hat{S}_{i j}\left(1+\zeta_{i j}\right)\left[\hat{H}_{i j, l}\left(1+\epsilon_{j}\right)\right]^{2} \delta \omega \delta \theta
$$

where index $l$ corresponds to the response of interest. Taking the variance of Eq.(13), where $S$ and $H_{l}$ are uncorrelated, leads to:

$$
\begin{aligned}
& \left(u_{R_{l}}\right)^{2}=\sum_{j=1}^{m} \sum_{i=1}^{n} \hat{S}_{i j}^{2} \hat{H}_{i j, l}^{4}(\delta \omega)^{2}(\delta \theta)^{2} \times \\
& \left(2 \sigma_{\epsilon_{j}}^{4}+4 \sigma_{\epsilon_{j}}^{2}+\sigma_{\zeta_{i j}}^{2}+2 \sigma_{\zeta_{i j}}^{2} \sigma_{\epsilon_{j}}^{4}+4 \sigma_{\zeta_{i j}}^{2} \sigma_{\epsilon_{j}}^{2}\right)
\end{aligned}
$$

This variability in the estimation of the variance of individual responses is presented here as the coefficient of variation of corresponding response which is defined by:

$$
C O V_{R_{l}}=\frac{u_{R_{l}}}{\hat{R}_{l}}
$$

where $\hat{R}_{l}$ is the estimated variance of the $l$ th response using Eq.(1). Table III shows this value for different responses at the considered wave cases in Table II. As seen in the table, the magnitudes are considerable except in case B. Specially, the uncertainty of roll estimation is quite large in case of stern quartering wind sea. 


\section{CONCLUSION}

The paper proposes a procedure to quantify the uncertainty of a method for estimation of wave spectra using a parametric wave model. Uncertainty analysis is carried out by propagating the errors in the inputs, which are transfer functions here, through the model for wave estimation, and all the way to the final output which is the variance of the expected short-term responses. This is implemented by taking both the wave spectrum and the transfer functions as random input variables to the response estimator. The standard deviations in the output represent the reliability measures of response predictions for the purpose of decision support onboard ships.

\section{ACKNOWLEDGMENT}

The authors sincerely acknowledge Class NK for supporting the research project in cooperation with the Technical University of Denmark.

\section{REFERENCES}

[1] U.D. Nielsen and T. Iseki. Prediction of FirstOrder Vessel Responses with Applications to Decision Support Systems. In 5th World Maritime Technology Conference, 2015.

[2] U.D. Nielsen and J.J. Jensen. A novel approach for navigational guidance of ships using onboard monitoring systems. Ocean Engineering, 38(2-3):444-455, February 2011.

[3] N. Montazeri, U.D. Nielsen, and J.J Jensen. Estimation of wind sea and swell using shipboard measurements-A refined parametric modelling approach. Applied Ocean Research (under review), 2015.

[4] J.J. Jensen and J. Capul. Extreme response predictions for jack-up units in second order stochastic waves by FORM. Probabilistic Engineering Mechanics, 21(4):330-337, October 2006.

[5] I E C BIPM, ILAC IFCC, IUPAC ISO, and OIML IUPAP. Evaluation of measurement dataguide to the expression of uncertainty in measurement, JCGM 100: 2008 GUM 1995 with minor corrections, 2008.

[6] W. Qiu, J. Sales Junior, D. Lee, H. Lie, V. Magarovskii, T. Mikami, J. Rousset, S. Sphaier, L. Tao, and X. Wang. Uncertainties related to predictions of loads and responses for ocean and offshore structures. Ocean Engineering, 86:58-67, 2014.

[7] M. Irvine, J. Longo, and F. Stern. Pitch and Heave Tests and Uncertainty Assessment for a Surface Combatant in Regular Head Waves. Journal of Ship Research, 52(2):146163, 2008.

[8] C. Guedes Soares. Effect of transfer function uncertainty on short-term ship responses. Ocean Engineering, 18(4):329-362, 1991.

[9] U.D. Nielsen. Response-based estimation of sea state parametersinfluence of filtering. Ocean Engineering, 34(13):1797-1810, September 2007.

[10] R. Pascoal and C. Guedes Soares. Kalman filtering of vessel motions for ocean wave directional spectrum estimation. Ocean Engineering, 36(6-7):477-488, May 2009.

[11] Alexandre N. Simos, Eduardo a. Tannuri, João V. Sparano, and Vinícius L.F. Matos. Estimating wave spectra from the motions of moored vessels: Experimental validation. Applied Ocean Research, 32(2):191-208, April 2010.

[12] C. Guedes Soares. Effect of spectral shape uncertainty in the short term wave-induced ship responses. Applied Ocean Research, 12(2):54-69, April 1990.

[13] Yonghwan Kim and Greg Hermansky. Uncertainties in seakeeping analysis and related loads and response procedures. Ocean Engineering, 86:68-81, 2014. 